\title{
Myxomycetes collection preserved in the Herbarium of the Federal University of Roraima (Brazil)
}

\author{
Laise de Holanda CAVALCANTI ${ }^{1 *}$, Eneida Jucene dos Santos CAVALCANTI ${ }^{2}$, David Itallo BARBOSA ${ }^{1}$, Leandro \\ de Almeida Neves Nepomuceno AGRA ${ }^{1}$, Andrea Carla Caldas BEZERRA ${ }^{1}$, Antônia Aurelice Aurélio COSTA ${ }^{1}$ \\ 1 Universidade Federal de Pernambuco, Departamento de Botânica, Laboratório de Myxomycetes, Av. Prof. Moraes Rego, s.n., Cidade Universitária, CEP: 50670-901, \\ Fone: 21267802. Recife, Pernambuco, Brasil. laise@pq.cnpq.br, daviditallo-bio@hotmail.com, nevesagra@gmail.com, ac_caldas@hotmail.com, licebio2002@gmail.com \\ * Autor Correspondente: laise@pq.cnpq.br \\ 2 Universidade Federal de Roraima, Centro de Estudo da Biodiversidade, Campus Paricarana. Av. Cap. Ene Garcez, n² 2413, Bairro Aeroporto, CEP: 69310-000. \\ Boa Vista, Roraima, Brasil. ejucene@gmail.com
}

\begin{abstract}
First records of myxomycetes in the North region of Brazil go back to the $19^{\text {th }}$ century. Nevertheless, the myxobiota of this region is still largely unexplored, with only 42 species recorded, distributed in 20 genera and seven families. The objectives of this paper were to characterize the Myxomycetes collection of the Herbarium of the Federal University of Roraima (UFRR) and to add new records for the myxobiota of this State. The collection holds specimens collected in fragments of Open Ombrophilous Forest, Seasonal Semi-deciduous Forest, Riparian Forest, deforested areas and urban home gardens in the state of Roraima. The 157 exsiccates were analyzed and identified or redetermined based on identification keys, descriptions and illustrations. The collection is in good conditions of preservation and includes all subclasses of Myxomycetes, $83 \%$ of its orders, $50 \%$ of its families, and 20 species. Trichiales, with one family, three genera and six species, represents $62 \%$ of all exsiccates. Cribraria aff. splendens, Metatrichia vesparia, Physarella oblonga, Stemonaria longa and Stemonitis splendens are new records for Roraima and Arcyria obvelata, Comatricha pulchella, Stemonitis pallida and Stemonitopsis aequalis are referred for the first time in the Northern Region, enlarging the knowledge of the Brazilian geographic distribution of these species.
\end{abstract}

KEYWORDS: Brazilian Amazonia, biodiversity, biological collections, Neotropics

\section{Coleção de Myxomycetes conservada no Herbário da Universidade Federal de Roraima}

\begin{abstract}
RESUMO
Os primeiros registros de mixomicetos na Região Norte do Brasil remontam ao Século XIX. Todavia, a mixobiota desta região está ainda muito pouco explorada, com apenas 42 espécies registradas, distribuídas em 20 gêneros e sete famílias. Os objetivos deste estudo foram caracterizar a coleção de Myxomycetes do Herbário da Universidade Federal de Roraima (UFRR) e adicionar novos registros para a mixobiota deste estado. A coleção contém espécimes coletados em fragmentos de Floresta Ombrófila Aberta, Floresta Estacional Semi-decidual, Mata Ciliar, áreas desmatadas e quintais urbanos no estado de Roraima. As 157 exsicatas foram analisadas e identificadas ou redeterminadas com base em chaves de identificação, descriçóes e ilustrações. A coleção encontra-se em boas condiçóes de conservação e inclui todas as subclasses de Myxomycetes, 83\% das ordens, 50\% das famílias e 20 espécies. A ordem Trichiales, com uma família, três gêneros e seis espécies, corresponde a $62 \%$ das exsicatas. Cribraria aff. splendens, Metatrichia vesparia, Physarella oblonga, Stemonaria longa e Stemonitis splendens são novos registros para o estado de Roraima e Arcyria obvelata, Comatricha pulchella, Stemonitis pallida e Stemonitopsis aequalis sáo referidas pela primeira vez para a Regiáo Norte, ampliando a distribuição geográfica conhecida para estas espécies no Brasil.
\end{abstract}

PALAVRAS-CHAVE: Amazônia brasileira, biodiversidade, coleçôes biológicas, Neotrópicos 


\section{INTRODUCTION}

The Northern Region of Brazil, where almost all of the Amazon's biome is distributed, corresponds to nearly $45 \%$ of the country's total land area. Myxomycetes were first collected in this region at the closing of the 19th century (Berkeley and Cooke 1876). However, its myxobiota is still largely unexplored with only 42 species recorded, distributed in 20 genera and seven families (Cavalcanti 2002; 2012). For the state of Roraima, 23 species of Myxomycetes, representing the three subclasses, $83 \%$ of the orders and $50 \%$ of the families, are listed by Cavalcanti et al. (1999).

Species of this group of organisms collected in the country's Northern Region are deposited in the following Brazilian herbaria: SP (Instituto de Botânica, state of São Paulo), located in the country's Southeast Region; UFP (Universidade Federal de Pernambuco), located in the Northeast Region; MG (Museu Paraense Emílio Goeldi), INPA (Instituto Nacional de Pesquisas da Amazônia), and UFRR (Universidade Federal de Roraima), located in the states of Pará, Amazonas and Roraima, in the Northern Region. The objectives of this study were to characterize the Myxomycetes collection of the UFRR Herbarium, with exsiccates collected from different phytophysiognomies, and to add new records for the myxobiota of the state of Roraima.

\section{MATERIALS AND METHODS}

The UFRR Herbarium, founded in 1997, adopts the BRAHMS System (Botanical Research and Herbarium Management System, version 5.52) to catalog and database its collection, in which 157 specimens of Myxomycetes are recorded. The information depicted on the labels of these exsiccata, all from the state of Roraima, include the following data: municipality, locality and type of environment, geographical coordinates, type of sporulation substrate, collection date, collector and identifier.

The level of conservation of the exsiccates was evaluated, in accordance to criteria described by Dela Cruz et al. (2009), with modifications (Table 1), and the known distribution of the species in the Brazilian Amazon were ascertained from specialized bibliography.

The specimens were analyzed and identified or revised with the keys, descriptions and illustrations of Martin and Alexopoulos (1969) and Poulain et al. (2011). The scientific names were updated and the nomenclature follows Lado (2012). All the collections are from the state of Roraima, collected by E.J. Santos-Cavalcanti, I.G. Silva, N.A. Gomes, R.C. Caleffi and L.A. Pessoni, during the years 2002 and 2003.
Table 1 - Criteria adopted to establish the level of conservation of Myxomycetes exsiccates of the UFRR Herbarium.

\begin{tabular}{cl}
\hline Level & \multicolumn{1}{c}{ Description } \\
\hline 1 & No traces of sporocarps \\
\hline 2 & $\begin{array}{l}\text { Exhibiting only stalks and/or hypothallus of the } \\
\text { sporocarps originally collected }\end{array}$ \\
& $\begin{array}{l}\text { Heavily damaged sporocarps, precluding } \\
\text { identification }\end{array}$ \\
\hline 4 & Exsiccates containing $\geq 2$ complete sporocarps \\
\hline 5 & $\begin{array}{l}\text { Exsiccates containing } \geq 80 \% \text { of its sporocarps in } \\
\text { good conservation }\end{array}$ \\
\hline
\end{tabular}

\section{RESULTS}

The specimens of Myxomycetes of the UFRR Herbarium were collected in the state of Roraima, $61 \%$ in the municipality of Boa Vista, within an environment of Semideciduous Seasonal Forest (Bamerindus Farm), a Riparian Forest within an area of Cerrado (Brazilian Savanna) of the Cauamé Campus, UFRR, and urban home gardens. The remaining specimens (39\%) were collected in the municipality of Iracema, in deforested areas and within an environment of Open Ombrophilous Forest of the Roxinho Waterfall.

The collection is a recent one and the oldest exsiccates were collected in January 2002. The specimens are packed in cardboard boxes with a label glued to the lid and $71 \%$ of them exhibited sporocarps that enable the identification of the species.

The revision undertaken showed that four exsiccates (UFRR 2304, UFRR 2312, UFRR 2321 and UFRR 2323) do not correspond to specimens of Myxomycetes. Forty five fit into levels 1, 2 and 3, and the species was not possible to be identified.

The three subclasses accepted by Martin et al. (1983), 83\% of the orders and $50 \%$ of the families are represented in this small collection, that contains 20 species, corresponding to $62,5 \%$ of those known up to this moment for the myxobiota of Roraima (Table 2).

The number of samples per species varies from 1 to 25 and the five species more commonly represented are Hemitrichia calyculata (Speg.) M.L. Farr, H. serpula (Scop.) Rostaf., Arcyria denudata (L.) Wettst., Ceratiomyxa fruticulosa (Mull.) T. Macbr. and Arcyria cinerea (Bull.) Pers. (Table 2). 
Table 2 - Genera and species of Myxomycetes recorded for the Amazonian biome and for the state of Roraima and its representation in the collection of the UFRR Herbarium. 1. New record for Roraima. 2. New record for the Northern Region. 3. Cavalcanti et al. 1999. 4. Cavalcanti 2002. 5. Cavalcanti 2012.

\begin{tabular}{|c|c|c|c|}
\hline Genera/Species & $\begin{array}{c}\text { Brazilian } \\
\text { Amazon }^{4,5}\end{array}$ & Roraima ${ }^{3,4}$ & $\begin{array}{l}\text { UFRR Herbarium } \\
\text { exsiccates }(n-)\end{array}$ \\
\hline \multicolumn{4}{|l|}{ Arcyria } \\
\hline A. cinerea (Bull.) Pers. & + & + & 10 \\
\hline A. denudata (L.) Wettst. & + & + & 14 \\
\hline A. obvelata (Oeder) Onsberg ${ }^{2}$ & + & + & 1 \\
\hline \multicolumn{4}{|l|}{ Ceratiomyxa } \\
\hline C. fruticulosa (0.F. Müll.) T. Macbr. & + & + & 11 \\
\hline C. morchella A.L. Welden & + & + & 0 \\
\hline C. sphaerosperma Boedijn & + & + & 0 \\
\hline \multicolumn{4}{|l|}{ Collaria } \\
\hline C. arcyrionema (Rostaf.) Nann.-Bremek. ex Lado & + & - & 0 \\
\hline \multicolumn{4}{|l|}{ Comatricha } \\
\hline C. nigra (Pers. ex J.F. Gmel.) J. Schröt. & + & - & 0 \\
\hline C. pulchella (C. Bab.) Rostaf. ${ }^{2}$ & + & + & 1 \\
\hline \multicolumn{4}{|l|}{ Craterium } \\
\hline C. leucocephalum (Pers. ex J.F. Gmel.) Ditmar & + & + & 2 \\
\hline C. paraguayense (Speg.) G. Lister & + & - & 0 \\
\hline \multicolumn{4}{|l|}{ Cribraria } \\
\hline C. cancellata (Batsch) Nann.-Bremek. & + & + & 0 \\
\hline C. intricata Schrad. & + & - & 0 \\
\hline C. languescens Rex & + & - & 0 \\
\hline C. microcarpa (Schrad.) Pers. & + & + & 0 \\
\hline C. aff. splendens (Schrad.)Pers. ${ }^{1}$ & + & + & 1 \\
\hline \multicolumn{4}{|l|}{ Diderma } \\
\hline D. effusum (Schwein.) Morgan & + & - & 0 \\
\hline \multicolumn{4}{|l|}{ Didymium } \\
\hline D. clavus (Alb. \& Schwein.) Rabenh. & + & + & 0 \\
\hline D. melanospermum (Pers.) T. Macbr. & + & - & 0 \\
\hline D. minus (Lister) Morgan & + & - & 0 \\
\hline D. nigripes (Link) Fr. & + & + & 0 \\
\hline \multicolumn{4}{|l|}{ Fuligo } \\
\hline F. septica (L.) F.H. Wigg. & + & + & 0 \\
\hline \multicolumn{4}{|l|}{ Hemitrichia } \\
\hline H. calyculata (Speg.) M.L. Farr & + & + & 25 \\
\hline H. serpula (Scop.) Rostaf. ex Lister & + & + & 23 \\
\hline \multicolumn{4}{|l|}{ Lycogala } \\
\hline L. epidendrum (L.) Fr. & + & + & 3 \\
\hline \multicolumn{4}{|l|}{ Metatrichia } \\
\hline M. vesparia (Batsch) Nann.-Bremek. ex G.W. Martin \& Alexop. ${ }^{1}$ & + & + & 5 \\
\hline \multicolumn{4}{|l|}{ Physarella } \\
\hline P. oblonga (Berk. \& M.A. Curtis) Morgan ${ }^{1}$ & + & + & 1 \\
\hline \multicolumn{4}{|l|}{ Physarum } \\
\hline P. cinereum (Batsch) Pers. & + & - & 0 \\
\hline P. melleum (Berk. \& Broome) Massee & + & - & 0 \\
\hline P. nicaraguense T. Macbr. & + & - & 0 \\
\hline P. nucleatum $\operatorname{Rex}$ & + & + & 0 \\
\hline P. pezizoideum (Jungh.) Pavill. \& Lagarde & + & + & 0 \\
\hline P. rigidum (G. Lister) G. Lister & + & - & 0 \\
\hline P. stellatum (Massee) G.W. Martin & + & - & 0 \\
\hline P. tenerum Rex & + & - & 0 \\
\hline P. viride (Bull.) Pers. & + & - & 0 \\
\hline
\end{tabular}


Table 2 - Continuation

\begin{tabular}{|c|c|c|c|}
\hline Genera/Species & $\begin{array}{c}\text { Brazilian } \\
\text { Amazon }^{4,5}\end{array}$ & Roraima ${ }^{3,4}$ & $\begin{array}{l}\text { UFRR Herbarium } \\
\text { exsiccates }\left(n^{0}\right)\end{array}$ \\
\hline \multicolumn{4}{|l|}{ Stemonaria } \\
\hline S. Ionga (Peck) Nann.-Bremek., R. Sharma \& Y. Yamam. ${ }^{1}$ & + & + & 1 \\
\hline \multicolumn{4}{|l|}{ Stemonitis } \\
\hline S. axifera (Bull.) T. Macbr. & + & + & 2 \\
\hline S. flavogenita E. Jahn & + & + & 1 \\
\hline S. fusca Roth & + & + & 4 \\
\hline S. pallida Wing. ${ }^{2}$ & + & + & 2 \\
\hline S. splendens Rostaf. ${ }^{1}$ & + & + & 3 \\
\hline \multicolumn{4}{|l|}{ Stemonitopsis } \\
\hline S. typhina (F.H. Wigg.) Nann.-Bremek. & + & + & 1 \\
\hline S. aequalis (Peck) Y. Yamam. ${ }^{2}$ & + & + & 1 \\
\hline \multicolumn{4}{|l|}{ Trichia } \\
\hline T. favoginea (Batsch) Pers. & + & + & 0 \\
\hline \multicolumn{4}{|l|}{ Tubifera } \\
\hline T. microsperma (Berk. \& M.A. Curtis) G.W. Martin & + & + & 0 \\
\hline
\end{tabular}

\section{List of species}

The 11 Ceratiomyxales exsiccates are well preserved (levels 4 and 5) and its single family, Ceratiomyxaceae, is represented by Ceratiomyxa fruticulosa (Table 2; Figure 1).

\section{Ceratiomyxa fruticulosa (O.F.Müll.) T.Macbr.}

Boa Vista, Cauamé Campus, Riparian Forest, on a decomposing tree trunk, $2^{\circ} 51^{\prime} 49^{\prime \prime} \mathrm{N}, 60^{\circ} 42^{\prime} 54^{\prime \prime} \mathrm{W}, 13 /$ IX/2002, UFRR 2103 (5); 14/XII/2002, UFRR 2108 (4); 20/V/2002, UFRR 2132 (4); 13/XI/2002, UFRR 2185 (5); $2^{\circ} 51^{\prime} 50^{\prime \prime} \mathrm{N}, 60^{\circ} 42^{\prime} 59^{\prime \prime} \mathrm{W}, 18 / \mathrm{IX} / 2002$, UFRR 2173 (5); UFRR 2174 (5). Iracema, Roxinho Waterfall, Open Ombrophilous Forest, on a decomposing tree trunk, $2^{\circ} 17^{\prime} 58^{\prime \prime} \mathrm{N}, 61^{\circ} 20^{\prime} 11^{\prime \prime} \mathrm{W}, 18 / \mathrm{XII} / 2002$, UFRR 2204 (5); $2^{\circ} 18^{\prime} 00^{\prime \prime} \mathrm{N}, 61^{\circ} 20^{\prime} 12^{\prime \prime} \mathrm{W}, 22 / \mathrm{I} / 2003$, UFRR 2105 (5); $2^{\circ} 18^{\prime} 04^{\prime \prime} \mathrm{N}, 61^{\circ} 20^{\prime} 15^{\prime \prime} \mathrm{W}, 21 / \mathrm{I} / 2002$, UFRR 2124 (5); on a palm tree petiole, UFRR 2122 (5); UFRR 2123 (5). Distribution in the North Region of Brazil: Amapá; Amazonas; Pará; Roraima (Farr 1985; Cavalcanti 2002; 2012).

The order Liceales is represented by two of its three families: Reticulariaceae, with three exsiccates, and Cribrariaceae, with two specimens.

\section{Cribraria aff. splendens (Schrad.) Pers.}

Boa Vista, Bamerindus Farm, Semi-deciduous Seasonal Forest, on a palm tree petiole, $3^{\circ} 03^{\prime} 33^{\prime \prime} \mathrm{N}, 60^{\circ} 51^{\prime} 58^{\prime \prime} \mathrm{W}, 24 /$ VII/2002, UFRR 2142 (4). Although many sporocarps were not in good conditions of conservation, two of them conserved the necessary structures for identification. Distribution in the North Region of Brazil: this is the first reference to the species.

\section{Cribraria sp.}

Iracema, Roxinho Waterfall, Open Ombrophilous Forest, on a decomposing tree trunk, $2^{\circ} 18^{\prime} 04^{\prime \prime} \mathrm{N}, 61^{\circ} 20^{\prime} 15^{\prime \prime} \mathrm{W}$, 18/XII/2002, UFRR 2117 (3).

It was not possible to confirm the identification of exsiccate UFRR 2117, as it was in level 3, remaining the original identification.

The exsiccates of the family Reticulariaceae were in good conditions of conservation and had their identities confirmed (Table 2; Figure 1).

\section{Lycogala epidendrum (L.) Fr.}

Boa Vista, Cauamé Campus, Riparian Forest, on a decomposing tree trunk, $2^{\circ} 51^{\prime} 49^{\prime \prime} \mathrm{N}, 60^{\circ} 42^{\prime} 54^{\prime \prime} \mathrm{W}, 14 /$ XII/2002, UFRR 2111 (4); UFRR 2112 (5); 13/XI/2002, UFRR 2187 (4). Distribution in the North Region of Brazil: Amazonas; Roraima (Cavalcanti et al. 1999).

Physarales, represented by three genera of the Physaraceae family, comprise 3\% of the collection. Two Physarum exsiccates were in level 2 of conservation and species identification was not possible. Three exsiccates in good conditions of conservation (level 4) were identified, commented as follows, with their corresponding levels of conservation (Figure 1).

\section{Craterium leucocephalum (Pers. ex J.F. Gmel.) Ditmar}

Boa Vista, Bamerindus Farm, Semi-deciduous Seasonal Forest, on decayed leaves, $2^{\circ} 59^{\prime} 32^{\prime \prime} \mathrm{N}, 60^{\circ} 52^{\prime} 48^{\prime \prime} \mathrm{W}, 18 /$ VI/2003, UFRR 2318 (4); UFRR 2319 (4). Distribution in the North Region of Brazil: Amazonas; Roraima (Cavalcanti et al. 1999). 


\section{Physarella oblonga (Berk. \& M.A.Curtis) Morgan}

Iracema, Roxinho Waterfall, Open Ombrophilous Forest, on a decomposed palm tree petiole, $2^{\circ} 16^{\prime} 50^{\prime \prime} \mathrm{N}$, 61'19'44' 'W, 21/VIII/2002, UFRR 2150 (4). Distribution in the North Region of Brazil: Amazonas; Pará (Cavalcanti 2012).

\section{Physarum spp.}

Boa Vista, Bamerindus Farm, Semi-deciduous Seasonal Forest, on a decomposing tree trunk, $2^{\circ} 59^{\prime} 38^{\prime \prime} \mathrm{N}$, 6052'48' 'W, 14/XII/2002, UFRR 2311 (2). Iracema, Roxinho Waterfall, Open Ombrophilous Forest, on a living tree trunk, $2^{\circ} 18^{\prime} 04^{\prime \prime} \mathrm{N}, 61^{\circ} 20^{\prime} 15^{\prime \prime} \mathrm{W}$, UFRR 2313(2).

Stemonitaceae, the only family of the order Stemonitales, is represented by 29 exsiccates ( $18 \%$ of the collection), originally identified on the level of genera, fitting all levels of conservation, $55 \%$ of them in levels 4 and 5 (Figure 1).

\section{Comatricha pulchella (C.Bab.) Rostaf.}

Iracema, Roxinho Waterfall, Open Ombrophilous Forest, on a palm tree petiole, $2^{\circ} 18^{\prime} 04^{\prime \prime} \mathrm{N}, 61^{\circ} 20^{\prime} 15^{\prime \prime} \mathrm{W}, 21 /$ XII/2002, UFRR 2120 (4). Distribution in the North Region of Brazil: this is the first reference to the species.

\section{Stemonaria longa (Peck.) Nann.-Bremek., R. Sharma \& Y. Yamam.}

Boa Vista, on a decomposing tree trunk, 2 $2^{\circ} 50^{\prime} 25.78^{\prime \prime} \mathrm{N}$, $60^{\circ} 42^{\prime} 27.49^{\prime \prime} \mathrm{W}, 30 / \mathrm{V} / 2003$, UFRR 2337 (4). Distribution in the North Region of Brazil: Amazonas (Damasceno et al. 2009).

\section{Stemonitis axifera (Bull.) T. Macbr.}

Boa Vista, Cauamé Campus, Riparian Forest, on a decomposing tree trunk, $2^{\circ} 51^{\prime} 49^{\prime \prime} \mathrm{N}, 60^{\circ} 42^{\prime} 54^{\prime \prime} \mathrm{W}$, 20/V/2002, UFRR 2096 (4). Iracema, Roxinho Waterfall, Open Ombrophilous Forest, on a decomposing tree trunk, $2^{\circ} 18^{\prime} 04^{\prime \prime} \mathrm{N}, 61^{\circ} 20^{\prime} 15^{\prime \prime} \mathrm{W}, 14 / \mathrm{IX} / 2002$, UFRR 2161 (4). Distribution in the North Region of Brazil: Amazonas; Roraima (Cavalcanti 2012).

\section{Stemonitis flavogenita E. Jahn}

Boa Vista, Cauamé Campus, Riparian Forest, on a decomposing tree trunk, 2 $51^{\prime} 49^{\prime \prime} \mathrm{N}, 60^{\circ} 42^{\prime} 54^{\prime \prime} \mathrm{W}$, 25/I/2002, UFRR 2126 (4). Distribution in the North Region of Brazil: Roraima (Cavalcanti et al. 1999).

\section{Stemonitis fusca Roth}

Boa Vista, Cauamé Campus, Riparian Forest, on a decomposing tree trunk, $2^{\circ} 51^{\prime} 49^{\prime \prime} \mathrm{N}, 60^{\circ} 42^{\prime} 54^{\prime \prime} \mathrm{W}, 13 /$ IX/2002, UFRR 2098 (4); UFRR 2105 (4); 25/III/2002, UFRR 2128 (5); Bamerindus Farm, Semi-deciduous Seasonal Forest, on a decomposing tree trunk, $3^{\circ} 03^{\prime} 33^{\prime \prime} \mathrm{N}$, $60^{\circ} 51^{\prime} 58^{\prime \prime} \mathrm{W}, 25 / \mathrm{VIII} / 2003$, UFRR 2152 (4). Distribution in the North Region of Brazil: Amazonas; Pará; Roraima (Cavalcanti 2002).

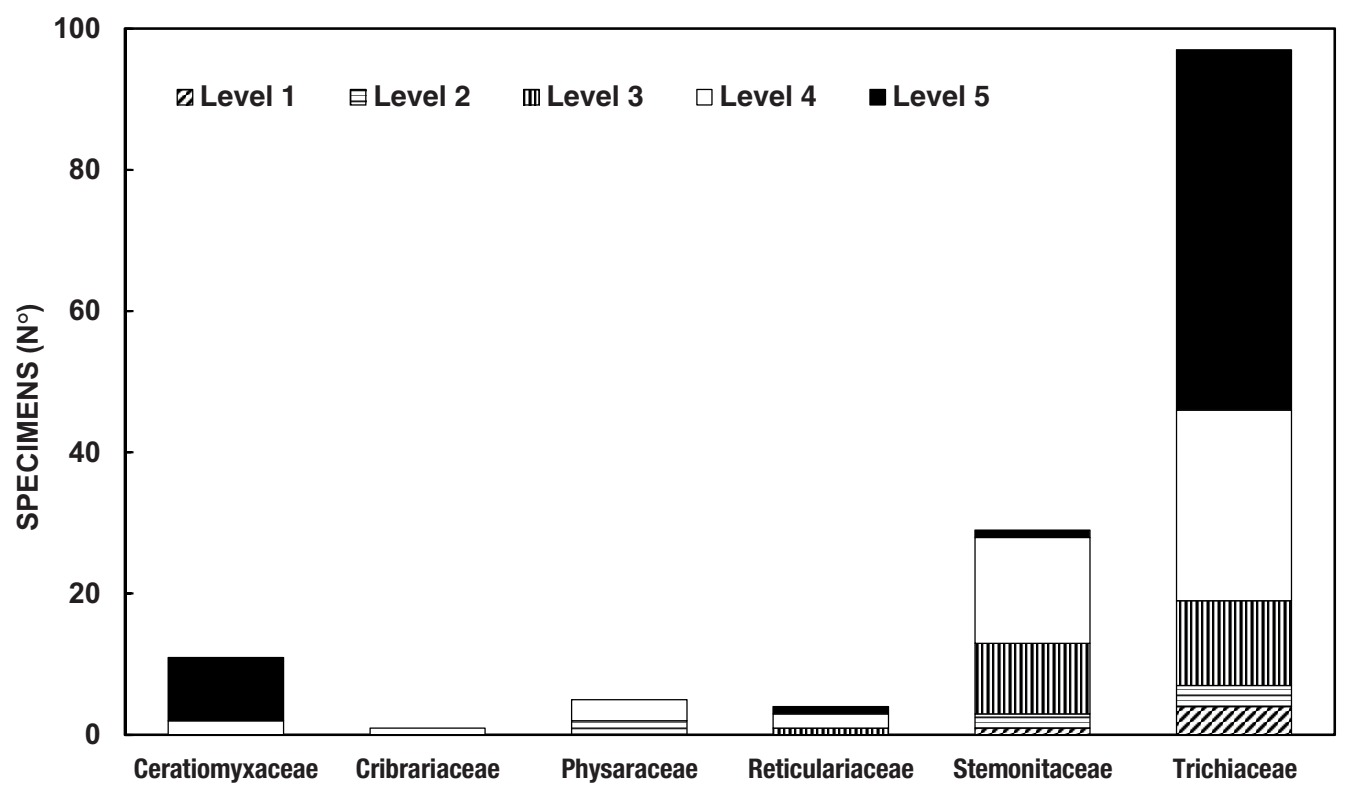

Figure 1 - Representative of the families in the Myxomycetes collection of the UFRR Herbarium and the levels of conservation of its exsiccates. Level 1: No traces of sporocarps. Level 2: Exhibiting only stalks and/or hypothallus of the sporocarps originally collected. Level 3: Heavily damaged sporocarps, precluding identification. Level 4: Exsiccates containing $\geq 2$ complete sporocarps. Level 5 : Exsiccates containing $\geq 80 \%$ of its sporocarps in good conservation. 


\section{Stemonitis pallida Wing.}

Boa Vista, on a decomposing tree trunk, $2^{\circ} 50^{\prime} 25.78^{\prime \prime} \mathrm{N}$, 6042'27.49' W, 30/V/2003, UFRR 2335 (4); Cauamé Campus, Riparian Forest, on a decomposing tree trunk, $2^{\circ} 51^{\prime} 49^{\prime \prime} \mathrm{N}, 60^{\circ} 42^{\prime} 54^{\prime \prime} \mathrm{W}, 14 / \mathrm{XII} / 2002$, UFRR 2107 (4). Distribution in the North Region of Brazil: this is the first reference to the species.

\section{Stemonitis splendens Rostaf.}

Boa Vista, Cauamé Campus, Riparian Forest, on a decomposing tree trunk, $2^{\circ} 51^{\prime} 49^{\prime \prime} \mathrm{N}, 60^{\circ} 42^{\prime} 54^{\prime \prime} \mathrm{W}, 13 /$ V2002, UFRR 2097 (4); Bamerindus Farm, Semi-deciduous Seasonal Forest, on a decomposing tree trunk, $2^{\circ} 59^{\prime} 32^{\prime \prime} \mathrm{N}$, 60 $59^{\prime} 48^{\prime \prime} \mathrm{W}, 18 / \mathrm{VI} / 2003$, UFRR 2322 (4). Iracema, Roxinho Waterfall, Open Ombrophilous Forest, on a living tree trunk, $2^{\circ} 18^{\prime} 04^{\prime \prime} \mathrm{N}, 61^{\circ} 20^{\prime} 15^{\prime \prime} \mathrm{W}, 27 / \mathrm{V} / 2003$, UFRR 2315 (4). Distribution in the North Region of Brazil: Amazonas; Pará (Cavalcanti et al. 1999).

\section{Stemonitopsis aequalis (Peck) Y. Yamam.}

Boa Vista, Cauamé Campus, Riparian Forest, on a decomposing tree trunk, $2^{\circ} 51^{\prime} 49^{\prime \prime} \mathrm{N}, 60^{\circ} 42^{\prime} 54^{\prime \prime} \mathrm{W}$, 25/I/2002, UFRR 2127 (4). Distribution in the North Region of Brazil: this is the first reference to the species.

\section{Stemonitopsis typhina (F.H.Wigg.) Nann.-Bremek.}

Boa Vista, Bamerindus Farm, Semi-deciduous Seasonal Forest, on a decomposing tree trunk, $3^{\circ} 03^{\prime} 33^{\prime \prime} \mathrm{N}$, $60^{\circ} 51^{\prime} 58^{\prime \prime} \mathrm{W}, 28 / \mathrm{VIII} / 2002$, UFRR 2157 (4). Distribution in the North Region of Brazil: Amazonas; Roraima (Cavalcanti et al. 1999, as Comatricha typhoides (Bull.) Rostaf.)

The Trichiales, represented by the Trichiaceae family, comprise $62 \%$ of the collection, with exsiccates fitting in all conservation levels (Figure 1). The majority $(80 \%)$ were in good conditions of conservation (levels 4 and 5). Three genera and six species of Trichiaceae were identified, commented as follows, with their corresponding exsiccates and levels of conservation.

\section{Arcyria cinerea (Bull.) Pers.}

Boa Vista, Cauamé Campus, Riparian Forest, on a decomposing tree trunk, $2^{\circ} 51^{\prime} 49^{\prime \prime} \mathrm{N}, 60^{\circ} 42^{\prime} 54^{\prime \prime} \mathrm{W}, 13 /$ IX/2002, UFRR 2104 (4); $2^{\circ} 51^{\prime} 50^{\prime \prime} \mathrm{N}, 60^{\circ} 42^{\prime} 59^{\prime \prime} \mathrm{W}$, 24/VIII/2002, UFRR 2151 (5); Bamerindus Farm, Semideciduous Seasonal Forest, on a decomposing tree trunk, $2^{\circ} 59^{\prime} 32^{\prime \prime} \mathrm{N}, 60^{\circ} 52^{\prime} 48^{\prime \prime} \mathrm{W}, 13 / \mathrm{VIII} / 2003$, UFRR 2329 (5); 24/VII/2002, UFRR 2140 (5); 303'33' $\mathrm{N}, 60^{\circ} 51^{\prime} 58^{\prime \prime} \mathrm{W}$, UFRR 2141 (4); 28/VIII/2002, UFRR 2155 (4). Iracema, Roxinho Waterfall, Open Ombrophilous Forest, on a decomposing tree trunk, $2^{\circ} 18^{\prime} 01^{\prime \prime} \mathrm{N}, 61^{\circ} 20^{\prime} 15^{\prime \prime} \mathrm{W}, 20 /$ VII/2002, UFRR 2135 (4); 14/IX/2002, UFRR 2168 (4); $2^{\circ} 18^{\prime} 04^{\prime \prime} \mathrm{N}, 61^{\circ} 20^{\prime} 15^{\prime \prime} \mathrm{W}$, UFRR 2160 (5); on a palm tree petiole, $2^{\circ} 18^{\prime} 00^{\prime \prime} \mathrm{N}, 61^{\circ} 20^{\prime} 15^{\prime \prime} \mathrm{W}$, UFRR 2170 (5). Distribution in the North Region of Brazil: Acre; Amazonas; Pará; Roraima (Berkeley and Cooke 1876; Jahn 1904; Cavalcanti 1970; Cavalcanti et al. 1999).

\section{Arcyria denudata (L.) Wettst.}

Boa Vista, Cauamé Campus, Riparian Forest, on a decomposing tree trunk, $2^{\circ} 51^{\prime} 49^{\prime \prime} \mathrm{N}, 60^{\circ} 42^{\prime} 54^{\prime \prime} \mathrm{W}, 13 /$ IX/2002, UFRR 2102 (5); 14/XII/2002, UFRR 2195 (5); UFRR 2199 (4); $2^{\circ} 51^{\prime} 40^{\prime \prime} \mathrm{N}, 60^{\circ} 42^{\prime} 50$ '”W, 25/I/2003, UFRR 2300 (5); Aeroporto district, on a decomposing tree trunk in a home garden, $2^{\circ} 50^{\prime} 25.78^{\prime \prime} \mathrm{N}, 60^{\circ} 42^{\prime} 27.49^{\prime \prime} \mathrm{W}$, 30/V/2003, UFRR 2338 (5); Bamerindus Farm, Semideciduous Seasonal Forest, on a pileus of a Basidiomycete, $2^{\circ} 59^{\prime} 32^{\prime \prime} \mathrm{N}, 60^{\circ} 52^{\prime} 48^{\prime \prime} \mathrm{W}, 21 / \mathrm{V} / 2003$, UFRR 2305 (5); on a decomposing tree trunk, UFRR 2306 (5); on Basidiomycete,

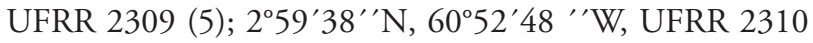
(5). Iracema, Roxinho Waterfall, Open Ombrophilous Forest, on a decomposing tree trunk, $2^{\circ} 18^{\prime} 00^{\prime \prime} \mathrm{N}, 61^{\circ} 20^{\prime} 12^{\prime \prime} \mathrm{W}$, 22/I/2003, UFRR 2206 (5); $2^{\circ} 18^{\prime} 00^{\prime \prime} \mathrm{N}, 61^{\circ} 20^{\prime} 15^{\prime \prime} \mathrm{W}$, 21/VIII/2002, UFRR 2146 (4); $2^{\circ} 18^{\prime} 01^{\prime \prime} \mathrm{N}, 61^{\circ} 20^{\prime} 12^{\prime \prime} \mathrm{W}$, 27/V/2003, UFRR 2317 (5); $2^{\circ} 18^{\prime} 04^{\prime \prime} \mathrm{N}, 61^{\circ} 20^{\prime} 15^{\prime \prime} \mathrm{W}$, UFRR 2314 (4); $2^{\circ} 17^{\prime} 58^{\prime \prime} \mathrm{N}, 61^{\circ} 20^{\prime} 11^{\prime \prime} \mathrm{W}, 18 / \mathrm{VIII} / 2003$, UFRR 2332 (4). Distribution in the North Region of Brazil: Amapá; Amazonas; Rondônia; Roraima (Farr 1985; Capelari and Maziero 1988; Cavalcanti et al. 1999; Cavalcanti 2002).

\section{Arcyria obvelata (Oeder) Onsberg}

Boa Vista, Cauamé Campus, Riparian Forest, on a decomposing tree trunk, $2^{\circ} 51^{\prime} 49^{\prime \prime} \mathrm{N}, 60^{\circ} 42^{\prime} 54^{\prime \prime} \mathrm{W}, 17 /$ VII/2002, UFRR 2133 (4). Distribution in the North Region of Brazil: this is the first reference to the species.

\section{Arcyria spp.}

Eleven Arcyria exsiccates were in the first three levels of conservation, and species identification was not possible: Boa Vista, Cauamé Campus, Riparian Forest, on a decomposing tree trunk, $2^{\circ} 51^{\prime} 49^{\prime \prime} \mathrm{N}, 60^{\circ} 42^{\prime} 54^{\prime \prime} \mathrm{W}, 20 / \mathrm{V} / 2002$, UFRR 2094 (3); 13/IX/2002, UFRR 2106 (2); 14/XII/20202, UFRR 2109 (1); UFRR 2110 (3); 13/XI/2002, UFRR 2188 (1); $2^{\circ} 51^{\prime} 50^{\prime \prime} \mathrm{N}, 60^{\circ} 42^{\prime} 59^{\prime \prime} \mathrm{W}, 20 / \mathrm{V} / 2002$, UFRR 2130 (3); Bamerindus Farm, Semi-deciduous Seasonal Forest, on a decomposing tree trunk, $02^{\circ} 59^{\prime} 32^{\prime \prime} \mathrm{N}, 60^{\circ} 52^{\prime} 48^{\prime \prime} \mathrm{W}, 24 /$

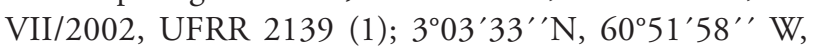
28/VIII/2002, UFRR 2154 (1) ; Aeroporto district, on a decomposing tree trunk in a home garden, $2^{\circ} 50^{\prime} 25.78^{\prime \prime} \mathrm{N}$, $60^{\circ} 42^{\prime} 27.49^{\prime \prime} \mathrm{W}, 30 / \mathrm{V} / 2003$, UFRR 2336 (2). Iracema, Roxinho Waterfall, Open Ombrophilous Forest, on a decomposing tree trunk, $2^{\circ} 17^{\prime} 58^{\prime \prime} \mathrm{N}, 61^{\circ} 20^{\prime} 11^{\prime \prime} \mathrm{W}, 14 /$ IX/2002, UFRR 2164 (3); on a decomposing tree trunk, $2^{\circ} 18^{\prime} 01^{\prime \prime} \mathrm{N}, 61^{\circ} 20^{\prime} 12^{\prime \prime} \mathrm{W}, 27 / \mathrm{V} / 2003$; UFRR 2316 (3). 


\section{Hemitrichia calyculata (Speg.) M.L. Farr}

Boa Vista, Cauamé Campus, Riparian Forest, on a decomposing tree trunk, $2^{\circ} 51^{\prime} 49^{\prime \prime} \mathrm{N}, 60^{\circ} 42^{\prime} 54^{\prime \prime} \mathrm{W}, 13 /$ IX/2002, UFRR 2099 (4); 20/V/2002, UFRR 2129 (5); UFRR 2131 (4); $2^{\circ} 51^{\prime} 50^{\prime \prime} \mathrm{N}, 60^{\circ} 42^{\prime} 59^{\prime \prime} \mathrm{W}, 18 / \mathrm{IX} / 2002$, UFRR 2171 (5); Bamerindus Farm, Semi-deciduous Seasonal Forest, on a decomposing tree trunk, $3^{\circ} 03^{\prime} 33^{\prime \prime} \mathrm{N}$, $60^{\circ} 51^{\prime} 58^{\prime \prime} \mathrm{W}, 28 / \mathrm{VIII} / 2002$, UFRR 2156 (4); UFRR 2158 (5); UFRR 2159 (4); 2 ${ }^{\circ} 59^{\prime} 32^{\prime \prime} \mathrm{N}, 60^{\circ} 52^{\prime} 48^{\prime \prime} \mathrm{W}, 21 / \mathrm{V} / 2003$, UFRR 2307 (5); 18/VI/2003, UFRR 2320 (4); UFRR 2324 (5). Iracema, Roxinho Waterfall, Open Ombrophilous Forest, on a decomposing tree trunk, $2^{\circ} 18^{\prime} 04^{\prime \prime} \mathrm{N}, 61^{\circ} 20^{\prime} 15^{\prime \prime} \mathrm{W}$, 18/XII/2002, UFRR 2113 (4); UFRR 2116 (4); 22/I/2003, UFRR 2295 (5); 1/VII/2003, UFRR 2326 (4); UFRR 2327 (4); 18/VIII/2003, UFRR 2330 (4); $2^{\circ} 18^{\prime} 07^{\prime \prime} \mathrm{N}$, $61^{\circ} 20^{\prime} 12^{\prime \prime} \mathrm{W}, 21 / \mathrm{I} / 2002$, UFRR 2125 (4); $2^{\circ} 18^{\prime} 00^{\prime \prime} \mathrm{N}$, $61^{\circ} 20^{\prime} 15^{\prime \prime} \mathrm{W}, 21 / \mathrm{VIII} / 2002$, UFRR 2147 (4); UFRR 2149 (4); $2^{\circ} 18^{\prime} 00^{\prime \prime} \mathrm{N}, 61^{\circ} 20^{\prime} 12^{\prime \prime} \mathrm{W}, 18 / \mathrm{XII} / 2002$, UFRR 2201 (4); 22/I/2003, UFRR 2299 (5); $2^{\circ} 18^{\prime} 01^{\prime \prime} \mathrm{N}, 61^{\circ} 20^{\prime} 15^{\prime \prime} \mathrm{W}$, 14/IX/2002, UFRR 2163 (4); UFRR 2166 (4); UFRR 2167 (4); $2^{\circ} 17^{\prime} 58^{\prime \prime} \mathrm{N}, 61^{\circ} 20^{\prime} 11^{\prime \prime} \mathrm{W}, 18 / \mathrm{XII} / 2002$, UFRR 2203 (5). Distribution in the North Region of Brazil: Amazonas; Roraima (Farr 1985; Cavalcanti et al. 1999; Cavalcanti 2002; Bezerra et al. 2009).

\section{Hemitrichia serpula (Scop.) Rostaf. ex Lister}

Boa Vista, Bamerindus Farm, Semi-deciduous Seasonal Forest, on a decomposing tree trunk, $3^{\circ} 03^{\prime} 33^{\prime \prime} \mathrm{N}$, $60^{\circ} 51^{\prime} 58^{\prime \prime} \mathrm{W}, 24 / \mathrm{IX} / 2002$, UFRR 2175 (5); UFRR 2176 (5); UFRR 2177 (5); UFRR 2178 (5); UFRR 2179 (5); UFRR 2180 (5); UFRR 2181 (5); UFRR 2182 (5); UFRR 2183 (5); UFRR 2184 (5); 11/XII/2002, UFRR 2190 (5); UFRR 2192 (5); UFRR 2193 (5); UFRR 2194 (5); $2^{\circ} 59^{\prime} 32^{\prime \prime} \mathrm{N}, 60^{\circ} 59^{\prime} 48^{\prime \prime} \mathrm{W}, 13 / \mathrm{VIII} / 2002$, UFRR 2328 (5). Iracema, Roxinho Waterfall, Open Ombrophilous Forest, on a decomposing tree trunk, $2^{\circ} 18^{\prime} 04^{\prime \prime} \mathrm{N}, 61^{\circ} 20^{\prime} 15^{\prime \prime} \mathrm{W}$, 21/XII/2002, UFRR 2119 (5); 22/I/2003, UFRR 2297 (5); UFRR 2298 (5); $2^{\circ} 18^{\prime} 01^{\prime \prime} \mathrm{N}, 61^{\circ} 20^{\prime} 15^{\prime \prime} \mathrm{W}, 20 / \mathrm{VII} / 2002$, UFRR 2138 (5); 14/IX/2002, UFRR 2165 (5); $2^{\circ} 16^{\prime} 50^{\prime \prime}{ }^{\prime} \mathrm{N}$, 61'19'44' 'W, 21/VIII/2002, UFRR 2144 (5); UFRR 2145 (5); $2^{\circ} 18^{\prime} 00^{\prime \prime} \mathrm{N}, 61^{\circ} 20^{\prime} 12^{\prime \prime} \mathrm{W}, 22 / \mathrm{I} / 2003$, UFRR 2296 (5). Distribution in the North Region of Brazil: Amapá; Amazonas; Pará; Roraima (Farr 1985; Cavalcanti et al. 1999; Cavalcanti 2012; Bezerra et al. 2009).

\section{Hemitrichia spp.}

Six Hemitrichia exsiccates were in levels 2 and 3 of conservation, where species identification was not possible: Boa Vista, Cauamé Campus, Riparian Forest, on a decomposing tree trunk, $2^{\circ} 51^{\prime} 49^{\prime \prime} \mathrm{N}, 60^{\circ} 42^{\prime} 54^{\prime \prime} \mathrm{W}, 13 / \mathrm{IX} / 2002$, UFRR
2100 (2); $2^{\circ} 51^{\prime} 50^{\prime \prime} \mathrm{N}, 60^{\circ} 42^{\prime} 59^{\prime \prime} \mathrm{W}, 25 / \mathrm{I} / 2003$, UFRR 2301 (3); Bamerindus Farm, Semi-deciduous Seasonal Forest, $3^{\circ} 03^{\prime} 33^{\prime \prime} \mathrm{N}, 60^{\circ} 51^{\prime} 58^{\prime \prime} \mathrm{W}, 24 / \mathrm{VII} / 2002$, UFRR 2143 (3); $2^{\circ} 59^{\prime} 32^{\prime \prime} \mathrm{N}, 60^{\circ} 52^{\prime} 48^{\prime \prime} \mathrm{W}, 21 / \mathrm{V} / 2003$, UFRR 2308 (3). Iracema, Roxinho Waterfall, Open Ombrophilous Forest, on a decomposing tree trunk, $2^{\circ} 18^{\prime} 04^{\prime \prime} \mathrm{N}, 61^{\circ} 20^{\prime} 15^{\prime \prime} \mathrm{W}$, 18/VIII/2003, UFRR 2331 (3); on dead wood, $2^{\circ} 17^{\prime} 58^{\prime \prime} \mathrm{N}$, 6120'11'”, UFRR 2333 (3).

\section{Metatrichia vesparia (Batsch) Nann.-Bremek. ex G.W. Martin \& Alexop.}

Boa Vista, Bamerindus Farm, Semi-deciduous Seasonal Forest, on a decomposing tree trunk, $3^{\circ} 03^{\prime} 33^{\prime \prime} \mathrm{N}$, $60^{\circ} 51^{\prime} 58^{\prime \prime} \mathrm{W}, 28 / \mathrm{VIII} / 2002$, UFRR 2153 (5); 11/ XII/2002, UFRR 2191 (5). Iracema, Roxinho Waterfall, Open Ombrophilous Forest, on a decomposing tree trunk, $2^{\circ} 18^{\prime} 04^{\prime \prime} \mathrm{N}, 61^{\circ} 20^{\prime} 15^{\prime \prime} \mathrm{W}, 18 / \mathrm{XII} / 2002$, UFRR 2115 (5); $2^{\circ} 16^{\prime} 50^{\prime \prime} \mathrm{N}, 61^{\circ} 19^{\prime} 44^{\prime \prime} \mathrm{W}, 21 / \mathrm{VIII} / 2002$, UFRR 2148 (5); $2^{\circ} 18^{\prime} 01^{\prime \prime} \mathrm{N}, 61^{\circ} 20^{\prime} 15^{\prime \prime} \mathrm{W}, 14 / \mathrm{IX} / 2002$, UFRR 2169 (5). Distribution in the North Region of Brazil: Amazonas (Farr 1985; Cavalcanti 2002; 2012). Roraima (this paper)

\section{DISCUSSION}

Myxomycetes specimens have been deposited in the UFRR Herbarium since 2002, by E. J. Santos-Cavalcanti and N. A. Gomes, all of them collected in the state of Roraima. Today, the collection includes species distributed in the subclasses Ceratiomyxomycetidae, Stemonitomycetidae and Myxogastromycetidae. Stemonitales and Trichiales have the highest number of genera and species.

The collection has not been reported in papers, and some genera and species are recorded for the first time to the Amazonian region or the Roraima state in the present revision. Based on the morphological characteristics of the sporocarps one can say that exsiccate UFRR 2142 is not Cribraria cancellata (Batsch.) Nann.-Bremek. neither C. microcarpa (Schrad.) Pers., referred by Cavalcanti et al. (1999) and Cavalcanti (2012) for Roraima or C. languescens Rex, referred by Cavalcanti (2012) for the Amazonian region. The characters with a taxonomic value related to the peridium and spores are similar to those of C. intricata Schrad. and C. splendens, that are connected by intermediary forms. In the material studied, the nodes from the peridium network do not exhibit the numerous typical free filaments of $C$. intricata, being therefore closer to C. splendens, that has a known distribution only in Pernambuco and São Paulo states (Hochgesand and Gottsberger 1996; Cavalcanti 2012). The only Arcyria obvelata exsiccate of the collection was found in good state of conservation and its sporocarps exhibited characteristics that are typical for the species, listed by Cavalcanti (2012) for the Northeastern (PE, PI, SE), Southeastern (SP), and Southern 
Regions (PR, SC), with no records for the Midwestern and Northern regions of the country. Metatrichia vesparia is widely distributed in Brazil, occurring in Atlantic and Amazon Forest environments (Cavalcanti 2012). Until now there were only records for the state of Amazonas, based on collections made by M. L. Farr in the municipality of Tapurucuará, in the vicinity of Rio Negro (Farr 1985).

\section{CONCLUSIONS}

The Myxomycetes collection of the UFRR Herbarium is in a good state of conservation and, although a small one, it contains almost all of the species known for the state of Roraima. The new records enlarge the knowledge of the myxobiota of the Brazilian Amazon, which is still little explored and makes the geographic distribution in Brazil of Arcyria obvelata, Comatricha pulchella, Cribraria splendens, Metatrichia vesparia, Physarella oblonga, Stemonaria longa, Stemonitis pallida, Stemonitis splendens and Stemonitopsis aequalis widely known.

\section{ACKNOWLEDGEMENTS}

The authors wish to thank the Conselho Nacional de Desenvolvimento Científico e Tecnológico (CNPq) for its financial support (Proc.150054/2011-4 PROTAX) and Dr. Albanita de Jesus Rodrigues da Silva, curator of the Herbarium of the Federal University of Roraima (UFRR) for allowing access to the collection and for the sending of microscope slides to the Myxomycetes Laboratory, Federal University of Pernambuco.

\section{REFERENCES}

Berkeley, M.J.; Cooke, M.C. 1876. The fungi of Brasil. Journal of the Linnean Society, 15: 363-398.

Bezerra, A.C.C.; Cavalcanti, L.H.; Dianese, J.C. 2009. Species of Hemitrichia (Trichiaceae, Myxomycetes) in Brazil. Mycotaxon, 107: $35-48$.

Capelari, M.; Maziero, R. 1988. Fungos macroscópicos do Estado de Rondônia, região dos rios Jaru e Ji-Paraná. Hohenea, 15: 28-36.

Cavalcanti, L.H. 1970. Coleção de Mixomicetos do Museu Paraense Emílio Goeldi. BoIetim do Museu Paraense Emílio Goeldi, Botânica, 35: 1-5.
Cavalcanti, L.H. 2002. Biodiversidade e distribuição de mixomicetos em ambientes naturais e antropogênicos no Brasil: espécies ocorrentes nas Regiôes Norte e Nordeste. In: Araujo, E.L.L.A.; Moura, A.N.; Sampaio, E.V.S.B.; Gestinari, L.M.S.; Carneiro, J.M.T. (Ed.). Biodiversidade, conservação e uso sustentável da flora do Brasil. Universidade Federal Rural de Pernambuco, Sociedade Botânica do Brasil, Recife, Pernambuco, p. 209-216.

Cavalcanti, L.H. 2012. Myxomycota. Lista de espécies da Flora do Brasil, Jardim Botânico do Rio de Janeiro. (www.floradobrasil. jbrj.gov.br/2012). Accessed on 12/04/2012.

Cavalcanti, L.H.; Santos, E.J.; Gomes, N.A. 1999. Myxomycetes do estado de Roraima, com especial referência a Estação Ecológica de Maracá (Amajari - RR, Brasil). Acta Amazonica, 29: 195-200.

Damasceno, G.; Costa, A.A.A; Passavante, Z.O; Cavalcanti, L.H. 2009. Stemonaria fuscoides (Stemonitaceae, Myxomycetes): A new reference for Brazil. Mycotaxon, 108: 205-211.

Dela Cruz, T.E.E; Kuhn, R.V.; Javier, A.O.M.; Parra, C.M.; Quimio, T.H. 2009. Status of the Myxomycetes collection at the UPLB, Museum of Natural History (UPLBMNH) Mycological Herbarium. Philippine. Journal of Systematic Biology, 3: 97-111.

Farr, M.L. 1985. Notes on Myxomycetes IV. Species collected in Brazil and Japan. Nova Hedwigia, 41: 167-175.

Hochgesand, E.; Gottsberger, G. 1996. Myxomycetes from the State of São Paulo, Brazil. Boletim do Instituto de Botânica, 10: 1-46.

Jahn, E. 1904. Myxomyceten aus Amazonas. Hedwigia, 43: 300 -305 .

Lado, C. 2012. An on-line nomenclatural information system of Eumycetozoa. (www.nomen.eumycetozoa.com). Accessed on $11 / 06 / 2012$.

Martin, G.W.; Alexopoulos, C.J. 1969. The Myxomycetes. University of Iowa Press, Iowa City, 561p.

Martin, G.W.; Alexopoulos, C.J.; Farr, M.L. 1983. The genera of Myxomycetes. University of Iowa Press, Iowa City, 198p.

Poulain, M.; Meyer, M.; Bozonnet, J. 2011. Les Myxomycètes. Fédération Mycologique et Botanique Dauphiné-Savoie, Sévrier, Guide de determination 568p, Planches 544p.

Recebido em 16/10/2012

Aceito em 22/04/2013 